\title{
Small molecule inhibitors for acute myeloid leukemia: where is the field heading?
}

\author{
"Given the poor landscape for acute myeloid leukemia therapeutic \\ development over the past decades, inhibition of these novel targets \\ could be poised to exert a paradigm shift in the treatment of patients \\ with this hematological malignancy."
}

First draft submitted: 8 May 2017; Accepted for publication: 11 May 2017; Published online: 10 August 2017

\section{Keywords: AKR1C3・AML・IDH1 • menin-MLL • RUNX1}

Acute myeloid leukemia (AML) is a hematologic neoplasm characterized by proliferation of poorly differentiated myeloid progenitor cells [1]. The neoplasm results from either de novo factors or as a side effect of chemotherapy for the treatment of other cancers; therapy-related AML $[2,3]$. The incidence rate among adults younger than 65 is $1.2 \%$. However, the neoplasm is more prevalent in the elderly with an incidence of 17.6 per 100,000 for those older than 65 , with a median age of onset of 67 [4]. A majority of patients relapse and die of the disease within 2 years of remission [5]. Survival rates in patients older than 65 at 5 years are just $6-12 \%$ [6]. AML accounts for $25 \%$ of acute leukemias in children, yet is responsible for $>50 \%$ of leukemia-related deaths.

Improvements in AML therapy have been limited and approximately, only $40-50 \%$ of adult patients with AML are cured [7]. Young $(<1$ year old) age $[8]$ and older age are widely recognized risk factors for one of the major causes of therapeutic failure in AML; that patients are not able to tolerate the toxicity associated with the most intensive chemotherapeutic treatment regimes, resulting in an increased rate of early death. The second major cause of therapeutic failure is relapse due to therapeutic resistance [9], which is particularly significant in acute promyelocytic leukemia $[10,11]$, a subtype of AML.

The incidence and mortality rate, potential for resistance, and limited therapeutic options combine to make the discovery of alternative chemotherapeutics critical for patients, particular infants and those older than 65 , diagnosed with AML. To date, the use of alternative chemotherapeutics in AML has been largely disappointing [4]. However, small molecule inhibitors of a number of recently identified protein targets offer new therapeutic options tailored to specific mutations or to counter resistance. In the rapidly growing field of precision (or genomic) medicine, the exact molecular aberrations present in individual patient samples can be determined and exploited for targeted therapy, resulting in greater therapeutic response [12]; an approach used to great effect in the clinical chemotherapeutic dasatinib which targets BCR-ABL tyrosine kinase with response rates well above $90 \%$ [13].

IDH acts to catalyze conversion of isocitrate to $\alpha$-ketoglutarate, regulating various epigenetic states. Mutations in IDH1 and IDH2 are mutually exclusive in AML, carried by approximately $10-40 \%$ of patients and result in gain of function to produce the oncometabolite 2-hydroxyglutarate (2HG), compounded with abrogation of normal function. Mutations in IDH1 promote cellular differentiation blockade and progression to leukemia by DNA and histone hypermethylation.

Okoye-Okafor et al. conducted a highthroughput screen and structural optimization to identify GSK321, an allosteric inhibitor of mutant IDH1 (Figure 1) [14]. This inhibitor possessed an $\mathrm{IC}_{50}$ of up to $2.9 \mathrm{nM}$ against the R132G mutation with $46 \mathrm{nM}$ against wt IDH1. Translation to primary R132G

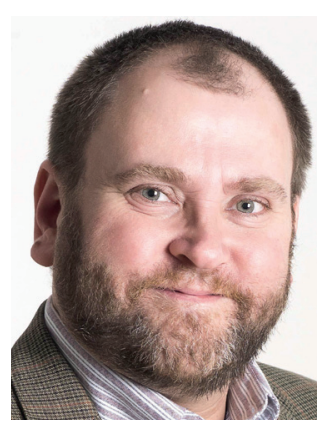

Paul C Trippier

Department of Pharmaceutical Sciences, Texas Tech University Health Sciences Center, School of Pharmacy, Amarillo, TX 79106, USA

and

Center for Chemical Biology, Department of Chemistry \& Biochemistry, Texas Tech University, Lubbock, TX 79409, USA paul.trippier@ttuhsc.edu 


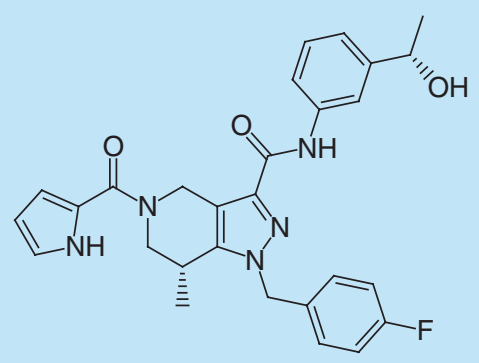

GSK321

Mutant IDH1 inhibitor

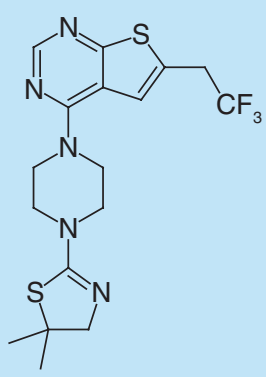

MI-2-2<smiles>CC(C)=CCc1ccc(/C=C/C(=O)O)cc1NC(=O)CCc1ccccc1</smiles>

KV37

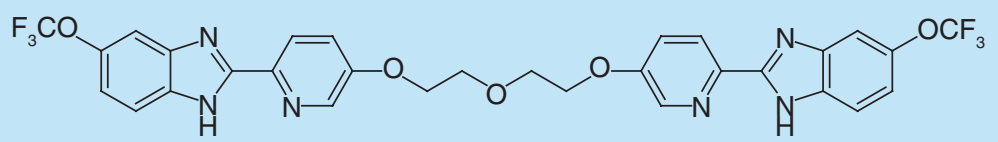

Al-10-49

CBFb-SMMHC-RUNX1 PPI inhibitor

Figure 1. Structures of representative small molecule inhibitors of acute myeloid leukemia targets discussed herein.

IDH1 AML cells was achieved, resulting in significant decrease in quiescent-phase cells and a dose-dependent decrease in 2HG levels coupled with no effect in healthy bone marrow. Further, approximately 7.4-fold induction of granulocytic differentiation was observed.

“

...drug development for acute myeloid leukemia therapeutics ... is shifting focus to inhibiting epigenetic events by protein-protein interaction blockade, targeting transcription factors and inhibiting metabolizing enzymes.

This study represents initial proof-of-concept that small molecule inhibition of mutant IDH1 in primary AML cells confers therapeutic effect. Such effect is multimodal, acting to diminish quiescent-phase cells and 2 HG levels as well as to promote differentiation. As AML is characterized by differentiation arrest, small molecules that act to induce differentiation of myeloid progenitor cells (differentiation therapy) would be therapeutic, and when coupled with the ability to modulate epigenetic changes, mutant IDH1 inhibitors represent a potentially powerful therapeutic strategy for patients harboring these mutations.

Chromosomal rearrangement of the $M L L$ gene occurs in $>70 \%$ of AML incidences in infants. The gene is responsible for the expression of MLL-fusion proteins. The protein menin directly interacts with all MLL-fusion proteins and is critical in controlling MLL-fusion protein-mediated transformation. Small molecule inhibition of this protein-protein interaction (PPI) has been shown to reverse MLL-fusion protein oncogenic activity [15]. Shi et al. described the identification of a potent small molecule inhibitor of the meninMLL PPI named MI-2-2 (Figure 1) [16]. This compound displayed an $\mathrm{IC}_{50}=46 \mathrm{nM}$ and provided significant cytotoxicity $\left(50 \%\right.$ growth inhibition $\left.\left[\mathrm{GI}_{50}\right]=0.2-1.2 \mu \mathrm{M}\right)$ in a panel of MLL-fusion transformed cells. The compound decreases $\mathrm{H} 3 \mathrm{~K} 4$ and $\mathrm{H} 3 \mathrm{~K} 79$ methylation in cell lines transformed with a variety of MLL-fusion proteins. Further, MI-2-2 markedly promoted monocytic differentiation. Treatment of primary cells from MLL leukemia patients resulted in substantial growth arrest.

While $M L L$ mutation occurs only in approximately $10 \%$ of all adult AML cases, in infants this mutation is detected in $>70 \%$ of cases. As targeting this protein results in significant therapeutic effect, both to reduce AML cell viability and to promote differentiation, this strategy may have particular success in the treatment of pediatric patients, where the current therapeutic landscape is severely limited.

The RUNX1 gene is the most frequent target for chromosomal translocation in leukemia. The transcription factor fusion CBFb-SMMHC (core binding factor $\beta$ and smooth-muscle myosin heavy chain) plays a key role in leukemia transformation. $\mathrm{CBFb}$ binds to RUNX proteins and the resulting complex is essential for normal hematopoiesis. CBFb-SMMHC outcompetes $\mathrm{CBFb}$ for binding to RUNX1, resulting in deregulated RUNX1 activity in hematopoiesis and induction of AML. Inhibitors of this complex are of particular interest as they represent early intervention to prevent platelet disorders progressing to AML. 
Employing a fluorescence resonance energy transfer assay, heteronuclear single quantum correlation nuclear magnetic resonance (HSQC NMR) techniques and medicinal chemistry, Illendula et al. [17] identified a small molecule PPI inhibitor of CBFb-SMMHC that disrupts binding to RUNX1. The lead inhibitor, AI-1049 (Figure 1) displayed an $\mathrm{IC}_{50}=260 \mathrm{nM}$ and selective ME-1 cell cytotoxicity $\left(\mathrm{IC}_{50}=0.6 \mathrm{mM}\right)$ over normal human bone marrow cells $\left(\mathrm{IC}_{50}=25 \mu \mathrm{M}\right)$. Furthermore, AI-10-49 selectively inhibits CBFb-SMMHC binding to RUNX1 over CBFb.

While this lead compound requires many years of additional work to possibly advance to the clinic, it does represent a proof-of-concept that targeting transcription factors in general and RUNX1 in particular, is possible and may open the door for additional scaffolds to progress toward treating patients before full development of AML. Targeting PPIs has been incredibly challenging to date; those small molecules that do succeed, continue to provide significant therapeutic effects, validating the search.

The ability of AKR1C3 to reduce prostaglandin $\mathrm{D}_{2}$ to $11 \beta-\mathrm{PGF}_{2 \alpha}$ and perform the reverse oxidation, accounts for the function of AKR1C3 as an important regulator of hematological malignant cell differentiation, proliferation and apoptosis, and hence potential therapeutic target for AML. Primary AML cell lines and several in vitro models predominantly express the AKR1C3 isoform. The action of AKR1C3 also results in the metabolism and inactivation of the anthracycline chemotherapeutics and decreases sensitivity to all-trans retinoic acid, contributing to the resistance of AML cells to these clinically employed agents.

Pharmacological inhibition of AKR1C3 holds the promise of a potentiation effect, sensitizing leukemia cells to the cytotoxic action of chemotherapeutics and resulting in an ability to counter resistance to etoposide, the anthracycline class, and other chemotherapeutics. The enzyme is however, a disputed target in the literature. A recent study reported that the specific AKR1C3 inhibitor 4-MDDT $\left(\mathrm{IC}_{50}=0.51 \mu \mathrm{M}\right)$ does not give the adjuvant effect at concentrations up to $50 \mu \mathrm{M}$ that a pan-AKR1C inhibitor does, despite the low expression of other isoforms in AML cells [18], thus requiring further credentialing of the target.

Work from within our own group has led to the development of highly potent and selective inhibitors of AKR1C3 [19]. The functionalized cinnamic acid derivative KV37 (Figure 1) demonstrates an AKR1C3 $\left(\mathrm{IC}_{50}=66 \mathrm{nM}\right)$ with 109-fold selectivity over the highly homologous AKR1C2 isoform. KV37 displays significant cytotoxicity to the AML HL-60 cell line at $50 \mu \mathrm{M}$. However, no effect is observed at concentrations below $1 \mu \mathrm{M}$. What is seen at these low concentrations is significant potentiation of the AML chemotherapeutics etoposide and daunorubcin. In pretreatment experiments, KV37 afforded six-fold potentiation of etoposide and ten-fold potentiation of daunorubicin. Low concentration treatment of KV37 resensitized daunorubicin-resistant AML cell lines to the cytotoxic action of the drug [20].

Therapeutic resistance and the inability to tolerate side effects of clinically approved AML chemotherapeutics are the main cause of morbidity and mortality in the disease. Development of potentiation agents that augment the action of chemotherapeutics results in widening of the therapeutic window. Small molecule inhibitors that act to inhibit enzymes involved in resistance mechanisms represent valuable synergistic agents to counter resistance development and a way to reactivate existing chemotherapeutics.

In summary, drug development for AML therapeutics, as in other forms of cancer, is shifting focus to inhibiting epigenetic events by PPI blockade, targeting transcription factors and inhibiting metabolizing enzymes. All of these targets have high prevalence of expression and/or mutation in AML cells over normal bone marrow, conveying selectivity. Development of multimodal small molecules that act to confer antineoplastic activity by multiple mechanisms of action are especially promising. Given the poor landscape for AML therapeutic development over the past decades, inhibition of these novel targets could be poised to exert a paradigm shift in the treatment of patients with this hematological malignancy.

Hematological cancers such as AML, by their nature, are particularly suited to precision medicine advances as a simple blood draw would allow characterization of specific molecular changes within the cancerous cells, foregoing the complicated and specialist biopsies required of solid tumors. Within hours, an AML patient could be determined to harbor specific mutations and start on a treatment regime of small molecule inhibitors designed to engage those specific mutated proteins.

\section{Financial \& competing interests disclosure}

The author is grateful to the organizations that have provided funding support for his group's research into AML drug discovery, including Leukemia Texas, The Cancer Prevention Research Institute of Texas (RP170003) and Texas Tech University Health Sciences Center School of Pharmacy. The author has no other relevant affiliations or financial involvement with any organization or entity with a financial interest in or financial conflict with the subject matter or materials discussed in the manuscript apart from those disclosed.

No writing assistance was utilized in the production of this manuscript. 


\section{References}

1 Shipley JL, Butera JN. Acute myelogenous leukemia. Exp. Hematol. 37(6), 649-658 (2009).

2 Kayser S, Dohner K, Krauter J et al. The impact of therapyrelated acute myeloid leukemia (AML) on outcome in 2853 adult patients with newly diagnosed AML. Blood 117(7), 2137-2145 (2011).

3 Patt DA, Duan Z, Fang S, Hortobagyi GN, Giordano SH. Acute myeloid leukemia after adjuvant breast cancer therapy in older women: understanding risk. J. Clin. Oncol. 25(25), 3871-3876 (2007).

4 Kuendgen A, Germing U. Emerging treatment strategies for acute myeloid leukemia (AML) in the elderly. Cancer Treat. Rev. 35(2), 97-120 (2009).

5 Farag SS, Ruppert AS, Mrozek K et al. Outcome of induction and postremission therapy in younger adults with acute myeloid leukemia with normal karyotype: a cancer and leukemia group B study. J. Clin. Oncol. 23(3), 482-493 (2005).

6 Kantarjian H, O'Brien S, Cortes J et al. Results of intensive chemotherapy in 998 patients age 65 years or older with acute myeloid leukemia or high-risk myelodysplastic syndrome: predictive prognostic models for outcome. Cancer 106(5), 1090-1098 (2006).

7 Meshinchi S, Arceci RJ. Prognostic factors and risk-based therapy in pediatric acute myeloid leukemia. Oncologist 12(3), 341-355 (2007).

8 Tomizawa D, Tawa A, Watanabe $\mathrm{T}$ et al. Appropriate dose reduction in induction therapy is essential for the treatment of infants with acute myeloid leukemia: a report from the Japanese Pediatric Leukemia/Lymphoma Study Group. Int . J. Hematol. 98(5), 578-588 (2013).

9 Estey E, Dohner H. Acute myeloid leukaemia. Lancet 368(9550), 1894-1907 (2006).

10 Coombs CC, Tavakkoli M, Tallman MS. Acute promyelocytic leukemia: where did we start, where are we now, and the future. Blood Cancer J. 5, e304 (2015).
11 Lehmann-Che J, Bally C, De The H. Resistance to therapy in acute promyelocytic leukemia. N. Engl. J. Med. 371(12), 1170-1172 (2014).

12 Turnbull AK. Personalized medicine in cancer: where are we today? Future Oncol. 11(20), 2795-2798 (2015).

13 Shah NP, Kim DW, Kantarjian $\mathrm{H}$ et al. Potent, transient inhibition of BCR-ABL with dasatinib $100 \mathrm{mg}$ daily achieves rapid and durable cytogenetic responses and high transformation-free survival rates in chronic phase chronic myeloid leukemia patients with resistance, suboptimal response or intolerance to imatinib. Haematologica 95(2), 232-240 (2010).

14 Okoye-Okafor UC, Bartholdy B, Cartier J et al. New IDH1 mutant inhibitors for treatment of acute myeloid leukemia. Nat. Chem. Biol. 11(11), 878-886 (2015).

15 He S, Malik B, Borkin D et al. Menin-MLL inhibitors block oncogenic transformation by MLL-fusion proteins in a fusion partner-independent manner. Leukemia 30 (2), 508-513 (2016).

16 Shi A, Murai MJ, He S et al. Structural insights into inhibition of the bivalent menin-MLL interaction by small molecules in leukemia. Blood 120(23), 4461-4469 (2012).

17 Illendula A, Pulikkan JA, Zong $\mathrm{H}$ et al. Chemical biology. A small-molecule inhibitor of the aberrant transcription factor CBFbeta-SMMHC delays leukemia in mice. Science 347(6223), 779-784 (2015).

18 Khanim F, Davies N, Velica P et al. Selective AKR1C3 inhibitors do not recapitulate the anti-leukaemic activities of the pan-AKR1C inhibitor medroxyprogesterone acetate. Br. J. Cancer 110(6), 1506-1516 (2014).

19 Zang T, Verma K, Chen M, Jin Y, Trippier PC, Penning TM. Screening baccharin analogs as selective inhibitors against type 5 17beta-hydroxysteroid dehydrogenase (AKR1C3). Chem. Biol. Interact. 234, 339-348 (2015).

20 Verma K, Zang T, Gupta N, Penning TM, Trippier PC. Selective AKR1C3 inhibitors potentiate chemotherapeutic activity in multiple acute myeloid leukemia (AML) cell lines. ACS Med. Chem. Lett. 7(8), 774-779 (2016). 